\title{
Drug retention of biological DMARD in rheumatoid arthritis patients: the role of baseline characteristics and disease evolution
}

\author{
Kim Lauper $\mathbb{D}^{1}$, Denis Mongin ${ }^{1}$, Deshire Alpizar-Rodriguez ${ }^{1}$, Catalin Codreanu², \\ Florenzo lannone $\mathbb{D}^{3}$, Eirik K. Kristianslund ${ }^{4}$, Tore K. Kvien ${ }^{4}$, Karel Pavelka ${ }^{5}$, \\ Manuel Pombo-Suarez ${ }^{6}$, Maria J. Santos ${ }^{7}{ }^{7}$, Cem Gabay ${ }^{1}$, Axel Finckh ${ }^{1}$ and \\ Delphine S. Courvoisier ${ }^{1}$
}

\begin{abstract}
Objective. To examine the association of the evolution in physician-reported and patient-reported outcomes with decision to stop biological DMARDs (bDMARDs) in RA. The contribution of baseline characteristics is well established, but little is known about how the disease evolution influences the decision to discontinue therapy.

Methods. RA patients who initiated a bDMARD treatment from 2009 and with information on date of visit were pooled from seven European RA registers. Each outcome was divided into baseline assessments (capturing the inter-individual differences at drug initiation) and changes from baseline at subsequent visits (capturing the individual evolution). Cox regression models were used to examine their association with drug discontinuation, adjusting for baseline patient and co-therapy characteristics and stratifying by register and calendar year of drug initiation.

Results. A total of 25077 patients initiated a bDMARDs (18507 a TNF-inhibitor, 3863 tocilizumab and 2707 abatacept) contributing an amount of 46456.8 patient-years. Overall, drug discontinuation was most strongly associated with a poor evolution of the DAS28, with a hazard ratio of $1.34(95 \% \mathrm{Cl} 1.29,1.40)$, followed by its baseline value. A change of Physician Global Assessment was the next strongest predictor of discontinuation, then the Patient Global Assessment.

Conclusions. The decision to discontinue treatments appears to be mostly influenced by DAS28 and particularly its evolution over time, followed by Physician Global Assessment evolution, suggesting that the decision to stop bDMARDs relies more on the physician's than on the patient's global assessment.
\end{abstract}

Key words: rheumatoid arthritis, biological therapies, DMARDs, tocilizumab, abatacept, tumour necrosis alpha inhibitors, time-varying, epidemiology

\section{Rheumatology key messages}

- Drug discontinuation was most strongly associated with the Disease Activity Score 28 (DAS28); mainly its evolution over time, followed by its baseline value.

- Patient global assessment was less associated with drug maintenance than disease activity and physician global assessment.

- The decision to stop bDMARDs relies mostly on the physician's assessment and appears not to take into account the patient's perception as much.

${ }^{1}$ Division of Rheumatology, University Hospitals Geneva, Geneva, Switzerland, ${ }^{2}$ Center of Rheumatic Diseases, University of Medicine and Pharmacy, Bucharest, Romania, ${ }^{3}$ GISEA, University Hospital of Bari, Bari, Italy, ${ }^{4}$ Department of Rheumatology, Diakonhjemmet Hospital, Oslo, Norway, Institute of Rheumatology, Prague and Clinic of Rheumatology, Charles University, Prague, Czech Republic, ${ }^{6}$ Rheumatology Unit, Clinical University Hospital, University of
Santiago de Compostela, Santiago, Spain and ${ }^{7}$ Rheumatology Department, Hospital Garcia de Orta, Almada, Portugal, on behalf of Reuma.pt

Submitted 30 January 2019; accepted 11 April 2019

Correspondence to: Kim Lauper, Division of Rheumatology, Geneva University Hospitals, 1211 Geneva 14, Switzerland.

E-mail: kim.lauper@hcuge.ch 


\section{Introduction}

Current recommendations for the management of RA advocate the early initiation of conventional synthetic DMARDs (csDMARDs) and a 'treat-to-target' strategy to achieve remission, with the objective being to prevent joint damage and disability, and to improve quality of life [1]. In patients with inadequate response, the addition of a biologic DMARD (bDMARD) or targeted synthetic DMARD is recommended to achieve better disease control [1]. Drug retention is often used as an outcome measure that integrates both the effectiveness and tolerance of the treatment [2]. However, the decision to discontinue medication remains largely subjective, when evaluating ineffectiveness or determining if the adverse event is severe enough. The decision may also be due to a combination of several reasons and other motives, such as patient preference, pregnancy, remission or a change of rheumatologist [3].

Many studies have focused on the association of baseline characteristics with drug retention of bDMARDs and have found female sex, higher baseline HAQ and absence of concomitant DMARDs to be associated with a higher risk of drug discontinuation [3, 4]. Such studies sometimes included time-varying variables $[5,6]$. In a study on RA patients from a US data bank, Ramiro et al. compared discontinuation rates of first and second bDMARD and sought to identify predictors of discontinuation using baseline and time-varying models [6]. Age, patient's global severity, comorbidity index, smoking status, cotherapy with MTX, mental and physical components of the Short Form 36, RA disease activity index, fibromyalgia criteria and pain were assessed once as baseline predictors and then as time-varying predictors. The authors found that time-varying predictors were similar to the baseline predictors, except for smoking and MTX co-therapy, which no longer predicted the outcome, and RA disease activity index, which became predictive of discontinuation. A study on the Swiss Rheumatic Diseases register of Gabay et al. looked at the effectiveness of bDMARDs in monotherapy vs in combination with csDMARDs in RA [5]. Seropositivity and the DAS28 were assessed as time-varying variables. In the first years of treatment, seropositivity and DAS28 were associated with higher bDMARDS discontinuation, but the effect decreased over time and became no longer significantly associated with discontinuation thereafter. However, these studies did not distinguish between the influence of the baseline disease characteristics of the patient and his or her evolution on drug retention.

Thus, the aim of this study is to examine the association of time-varying disease outcome characteristics with drug retention for bDMARDs in RA patients and their influence compared with their baseline characteristics.

\section{Methods}

PANABA and TOCERRA are two international collaborative projects, with patients on abatacept (ABA) and TNFinhibitors (TNFi) for PANABA, and tocilizumab (TCZ) and
TNFi for TOCERRA $[7,8]$. This study included the seven registers participating in both collaborations (Czech Republic, Italy, Norway, Portugal, Spain, Switzerland and Romania). Data are collected prospectively in the registers after the patient's inclusion. In order to be included in the registers, patients need to have a diagnosis of RA made by a rheumatologist and generally to be treated with a bDMARDs. However, for this study, only patients who had a visit entered in the registries at initiation of their bDMARDs were included. Patients could be included at their first bDMARDs and/or in later courses. To avoid duplicates, patients with TNFi were collected only from the TOCERRA registers. The local ethics committee approved the use of anonymized data for each register, and the Geneva Ethics Committee approved this collaboration study. Inclusion criteria were a diagnosis of RA by the treating physician, initiation of a bDMARD treatment and information on date of visit.

\section{Exposure of interests}

The time-varying variables of interest were the DAS28ESR or, if not available, the DAS28-CRP [with the Patient Global Assesment (PGA) included when possible (DAS28-4v)], the Clinical Disease Activity Index (CDAl), the HAQ Disability Index, the PGA, the Physician Global Assessment (PhGA) and the CRP. Each time-varying variable was divided into baseline assessments and changes from baseline at subsequent visits. The baseline values capture the inter-individual patients' differences at drug initiation, while the changes over time inform on the intra-individual evolution of the variables of interest (i.e. evolution of the patient between visits). All available visits were included. These covariates were chosen as exposures of interest a priori based on existing literature and clinical knowledge as the most likely to influence the choice to continue a drug or not. We chose to model the change between baseline and follow-up instead of the change between visits in the main analysis, as we think that looking at the difference between baseline and follow-up is more appropriate because small differences in between visits are likely to be less relevant on the decision to stop a drug than the general direction of the disease since treatment initiation.

\section{Study outcomes}

The main outcome was drug retention, which was defined as the time from the start date of drug treatment until the treatment discontinuation date plus one month, as we estimated that the patients were still under treatment during this time. If patients did not discontinue treatment, retention was censored at the date of the last reported follow-up visit plus one month. We also looked separately at discontinuation for intolerance and for ineffectiveness reasons as reported in the registers.

\section{Covariates}

The baseline covariates considered were age, gender, education, smoking status, disease duration, seropositivity, number of previously used bDMARDs, presence and 
type of concomitant csDMARDs, presence of glucocorticoids, year of treatment initiation and country of register. Seropositivity was defined as positive if RF and/or ACPA were positive, negative if both were negative and missing if one was missing and the other was negative to limit misclassification of exposure and assign seronegative status to patients with missing data. We also included as baseline covariates the presence of a cardiovascular comorbidity, as it has been associated with increased hazards of retention for abatacept [9] and the difference between tender joint count and swollen joint count on 28 joints ( $\triangle \mathrm{TSJ}$ ) as a study suggested that a discordance between tender joint count and swollen joint count may be associated with a reduced likelihood of remission [10]. These covariates were assessed at baseline and not considered as time-varying co-variates.

\section{Statistical methods}

We analysed baseline characteristics using standard descriptive statistics. Crude median drug retention was analysed with a Kaplan-Meier estimator. We used timevarying Cox regression models to examine the association of the baseline assessment and change over time of timevarying characteristics with drug discontinuation, using a strata term for country and year of treatment initiation and a cluster term to account for multiple treatment courses. To evaluate the relative importance of the time-varying variables, they were standardized as multiples of the standard deviation of the change. Missing covariates were imputed using multiple imputations with chained equations. Absence of significant collinearity was verified using variance inflation factor. All analyses and tabulations were performed using R v.3.4.2 with the tableone, mice, Hmisc and survival packages.

Two additional sensitivity analyses were performed. First, we looked at the effect of using different forms of DAS28 computation by running the analysis with the four different types of DAS28 calculations (DAS28-ESR and DAS28-CRP with or without PGA separately) instead of the composite DAS28. Second, we looked at the effect of changes in between visits instead of differences between follow-up and baseline values for the exposures of interest.

\section{Results}

\section{Patient characteristics}

A total of 25077 RA patients were included, contributing 46456.8 patient-years, of which $18507 \mathrm{TNFi}, 3863 \mathrm{TCZ}$ and 2707 ABA patients (Table 1, Supplementary Table S1, available at Rheumatology online). From those, 11078 stopped their treatment during follow-up (8199 TNFi, 1796 TCZ and 1083 ABA). Patients had a median age of 54.9 years (interquartile range $45.1,63.5$ ), median RA duration at bDMARDs initiation of 9.6 years (interquartile range $4.9,16.6$ ), median baseline DAS28 of 4.3 (interquartile range $3.6,5.6$ ) and were more often in co-therapy with a csDMARD than in monotherapy (78.3\%, Table 1). Patients with TNFi and TCZ were younger, with longer disease duration than ABA patients (Supplementary Table S1, available at Rheumatology online). TNFi patients were more often bio-naive (63.6\%) than TCZ (34.5\%) and ABA (35.1\%) patients. Patients with TCZ were more often in monotherapy (36.4\%) than patients with TNFi (18.8\%) or ABA (18.6\%). Patients with TCZ and ABA had higher DAS28, tender joint count, swollen joint count, PGA, PhGA, ESR and CRP than patients with TNFi.

\section{Drug discontinuation}

Overall crude median time to discontinuation was 2.9 years $(95 \% \mathrm{Cl} 2.8,3.0)$ with 2.9 years $(95 \% \mathrm{Cl} 2.8,3.0)$ for TNFi, 3.0 years $(95 \% \mathrm{Cl} 2.8,3.1)$ for TCZ and 2.7 years $(95 \% \mathrm{Cl}$ $2.4,2.9)$ for ABA. In the Cox multivariable model, for timevarying characteristics, discontinuation for all bDMARDs was most strongly associated with a poor evolution of DAS28, but not with CDAl (Table 2). However, when DAS28 was not entered in the models, change in CDAI was the strongest predictor of discontinuation for all bDMARDs (data not show). The change over time (intraindividual change) of DAS28 was a stronger predictor of discontinuation than the baseline values (inter-individual differences) (Fig. 1). The unstandardized hazard ratio (HR) was of $1.34(95 \% \mathrm{Cl} 1.29,1.40)$ for the evolution and of 1.29 $(95 \% \mathrm{Cl} 1.24,1.34)$ for the baseline value for each point of DAS28. Change of PhGA was the third strongest predictor of discontinuation. This order of importance (first evolution of DAS28, then baseline value, and finally change in PhGA) was similar for all three bDMARDs (Supplementary Fig. S2, available at Rheumatology online). There was only one exception to these global results. For ABA, PhGA change was more strongly associated with discontinuation than baseline DAS28. CRP and PGA change and baseline values were also associated with discontinuation in the whole group, but remained significant only for TNFi in the drug subgroups analyses. HAQ was the last significant predictor and was no longer significant in the subgroups analyses.

For the other variables for which the model was adjusted, younger age, female gender, shorter disease duration, higher number of previous bDMARDs, absence of concomitant csDMARDs, glucocorticoids at baseline, smoking and $\triangle T S J$ were associated with greater discontinuation in the whole population (Table 2). We did not find any association of cardiovascular comorbidity, seropositivity and education with drug survival. The trends were similar in the subgroups analyses although fewer variables reached statistical significance (Supplementary Table S2, available at Rheumatology online).

The results were similar when looking specifically at patients who stopped for adverse events or ineffectiveness, although the impact of time-varying factors was much less pronounced for patients who discontinued treatments for safety reasons compared with patients discontinuing for effectiveness reasons (data not shown).

Most of the patients had a DAS28-ESR with PGA (84\%), 9\% DAS28-ESR without PGA, 3\% DAS28-CRP with PGA and 4\% DAS28-CRP without PGA. In the first sensitivity analysis, using different forms of DAS28 calculation, we found similar results with DAS28 change and baseline 
TABLE 1 Sample characteristics at baseline

n

Total patient-years

Total number of visits (median [IQR])

Number of visits (\%)

1

2

$\geqslant 4$

Age, years (median [IQR])

Female gender (\%)

Education category (\%)

$0-10$ years

$11-13$ years

$>13$ years

Ever smoking (\%)

Cardiovascular comorbidity (\%)

Disease duration, years (median [IQR])

Seropositivity (\%)

Glucocorticoids (\%)

Glucocorticoid dose, mg/day (median [IQR])

Previous bDMARDs (\%)

0

1

2

$\geqslant 3$

Concomitant csDMARD (\%)

None

MTX

MTX and other

Other

DAS28 (median [IQR])

CDAI (median [IQR])

HAQ-DI (median [IQR])

TJC (median [IQR])

SJC (median [IQR])

$\triangle T S J$ (median [IQR])

PGA (median [IQR])

PhGA (median [IQR])

ESR $\mathrm{mm} / \mathrm{h}$ (median [IQR])

CRP mg/l (median [IQR])
46456.8

$3[2,6]$

\author{
$5556(22.2)$ \\ 4764 (19.0) \\ $3472(13.8)$ \\ $11285(45.0)$ \\ $54.9[45.1,63.5]$ \\ 19201 (76.6)
}

$4(0.02 \%)$

$7(0.03 \%)$

2889 (33.1)

$4326(49.6)$

1505 (17.3)

5913 (38.6)

$7149(45.7)$

$9.6[4.9,16.6]$

$11069(77.5)$

$10548(60.2)$

$5.0[5.0,10.0]$

16357 (65.23\%)

$$
\begin{array}{r}
13543(55.9) \\
6954(28.7) \\
2573(10.6) \\
1144(4.7)
\end{array}
$$

$$
\begin{gathered}
3852(21.7) \\
6620(37.3) \\
5769(32.5) \\
1511(8.5) \\
4.3[3.6,5.6] \\
17.6[13.5,24.7] \\
1.0[0.8,1.5] \\
6.0[2.0,12.0] \\
4.0[1.0,8.0] \\
1.0[0.0,4.0] \\
57.0[29.0,76.0] \\
40.0[17.0,60.0] \\
24.0[12.0,40.0] \\
4.3[1.0,13.9]
\end{gathered}
$$

9749 (38.88\%)

$9433(37.62 \%)$

$3150(12.56 \%)$

$10803(43.08 \%)$

7566 (30.17\%)

$19115(76.23 \%)$

863 (3.44\%)

7325 (29.21\%)

$0(0.00 \%)$

$0(0.00 \%)$

$0(0.00 \%)$

$5232(20.86 \%)$

5241 (20.90\%)

$6693(26.69 \%)$

8517 (33.96\%)

$5385(21.47 \%)$

$5022(20.03 \%)$

$0(0.00 \%)$

$\triangle T S J$ : difference between tender join count and swollen joint count on 28 joints; bDMARD: biological DMARD; CDAI: Clinical Disease Activity Index; DAS28: DAS 28 joints; csDMARD: conventional synthetic DMARD; HAQ-DI: HAQ-Disability index; IQR: interquartile range; PhGA: physician global assessment; PGA: patient global assessment; SJC: swollen joint counts on 28 joints; TJC: tender joint counts on 28 joints.

DAS28 being still the most influential variables on drug survival (Supplementary Table S3, available at Rheumatology online). However, when using DAS28 forms not including the PGA, PGA became proportionally more influential on drug retention, reaching almost a similar standardized hazards ratio as PhGA. For the second sensitivity analysis, we modelled change in between two consecutive visits instead of change between baseline and follow-up for the exposures of interests, and found that the standardized hazard ratios were overall smaller for each of the exposures of interests (Supplementary Table S4, available at Rheumatology online). DAS28 remained the exposure influencing drug survival most heavily, more than CDAl, although baseline DAS28 and change in DAS28 had about the same effect. After disease activity scores (DAS28 and CDAI), the exposures affecting drug discontinuation most heavily were $\mathrm{HAQ}$ change and $\mathrm{HAQ}$ baseline value, PhGA change and CRP change.

\section{Discussion}

To our knowledge, this study is one of the first to distinguish between the influence of the patient's baseline 
TABLE 2 Multivariable Cox model of discontinuing biological DMARDs stratified by country of register and year of treatment initiation and adjusted for drug treatment (tocilizumab, abatacept or TNF-inhibitors)

HR

Exposures of interest

$H A Q-D I^{a}$

DAS28 ${ }^{\mathrm{a}}$

$\mathrm{CDAl}^{\mathrm{a}}$

$\mathrm{PhGA}^{\mathrm{a}}$

$P G A^{a}$

$\mathrm{CRP}^{\mathrm{a}}$

Other variables for which the model was adjusted

Age in decade

Female gender

Education category (ref: $0-10$ years)

$11-13$ years

$>13$ years

Ever smoking

Cardiovascular comorbidity at baseline

Disease duration at baseline

Seropositivity

Glucocorticoids at baseline

Past bDMARDs (ref: none)

1

2

3 or more

Concomitant csDMARDs (ref: none)

MTX

MTX + other

Other

$\Delta$ TSJ

$95 \% \mathrm{CI}$

$\boldsymbol{P}$

\begin{tabular}{|c|c|c|}
\hline 1.03 & $1.00,1.07$ & 0.047 \\
\hline 1.05 & $1.01,1.09$ & 0.02 \\
\hline 1.51 & $1.42,1.61$ & $<0.001$ \\
\hline 1.67 & $1.55,1.79$ & $<0.001$ \\
\hline 1.00 & $0.94,1.06$ & 0.98 \\
\hline 1.05 & $0.97,1.13$ & 0.22 \\
\hline 1.12 & $1.08,1.17$ & $<0.001$ \\
\hline 1.18 & $1.13,1.23$ & $<0.001$ \\
\hline 1.05 & $1.01,1.09$ & 0.009 \\
\hline 1.07 & $1.03,1.12$ & $<0.001$ \\
\hline 1.05 & $1.02,1.08$ & 0.002 \\
\hline 1.08 & $1.05,1.12$ & $<0.001$ \\
\hline 0.96 & $0.94,0.98$ & $<0.001$ \\
\hline 1.07 & $1.02,1.13$ & 0.01 \\
\hline 0.99 & $0.91,1.07$ & 0.73 \\
\hline 1.00 & $0.90,1.12$ & 0.97 \\
\hline 1.07 & $1.02,1.13$ & 0.008 \\
\hline 1.04 & $0.99,1.10$ & 0.10 \\
\hline 0.99 & $0.99,0.99$ & $<0.001$ \\
\hline 0.94 & $0.89,1.00$ & 0.051 \\
\hline 1.17 & $1.10,1.24$ & $<0.001$ \\
\hline 1.18 & $1.13,1.24$ & $<0.001$ \\
\hline 1.34 & $1.25,1.44$ & $<0.001$ \\
\hline 1.34 & $1.21,1.49$ & $<0.001$ \\
\hline 0.86 & $0.80,0.92$ & $<0.001$ \\
\hline 0.88 & $0.81,0.96$ & 0.005 \\
\hline 0.94 & $0.87,1.01$ & 0.11 \\
\hline 1.00 & $0.99,1.00$ & 0.046 \\
\hline
\end{tabular}

The analysis is stratified by country of register and year of treatment initiation and adjusted for drug treatment (tocilizumab,

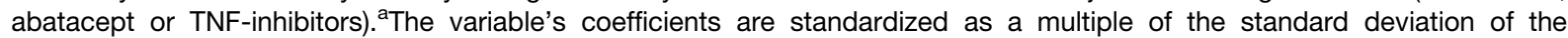
change. $\triangle \mathrm{TSJ}$ : difference between tender and swollen joint counts on 28 joints; ABA: abatacept; bDMARD: biological DMARD; CDAl: Clinical Disease Activity Index; DAS28: DAS 28 joints; csDMARD: conventional synthetic DMARD; HAQ-DI: HAQ-Disability Index; HR: hazard ratio; PhGA: physician global assessment; PGA: patient global assessment; TCZ: tocilizumab; TNFi: TNF inhibitors.

disease characteristics and his or her evolution on drug retention.

For most time-varying characteristics, the change over time (intra-individual evolution) was a stronger predictor of discontinuation than the baseline values (inter-individual differences). For time-varying variables, we found that the strongest effect on drug retention was associated with change of disease activity (DAS28 or CDAl). This was similar when looking specifically at patients for which the main stopping reason mentioned was ineffectiveness, but also safety. This result suggests that most physicians follow current recommendations to use clinical outcome measures to assess disease activity and adapt therapy [1]. Of note, change of CRP was a predictor of drug discontinuation only for TNFi. However, DAS28, a composite score that includes inflammatory markers, was a stronger predictor of discontinuation than CDAl. This finding was true even for TCZ, although it is considered an unsuitable indicator of disease activity considering the direct effect of TCZ on inflammatory markers [11].

PhGA change was the second strongest predictor of discontinuation, which implies that the decision to discontinue bDMARDs seems to be based more on the physician's assessment of disease activity than on patient perception. This finding raises questions regarding shared decision making of treatment discontinuation. Current adherence to bDMARDs varies between 32\% and $89 \%$ depending on the study setting $[12,13]$. As more and more DMARDs are self-administered orally or 
FIG. 1 Standardized hazard ratios of drug discontinuation for all biological DMARDS by time-varying variables

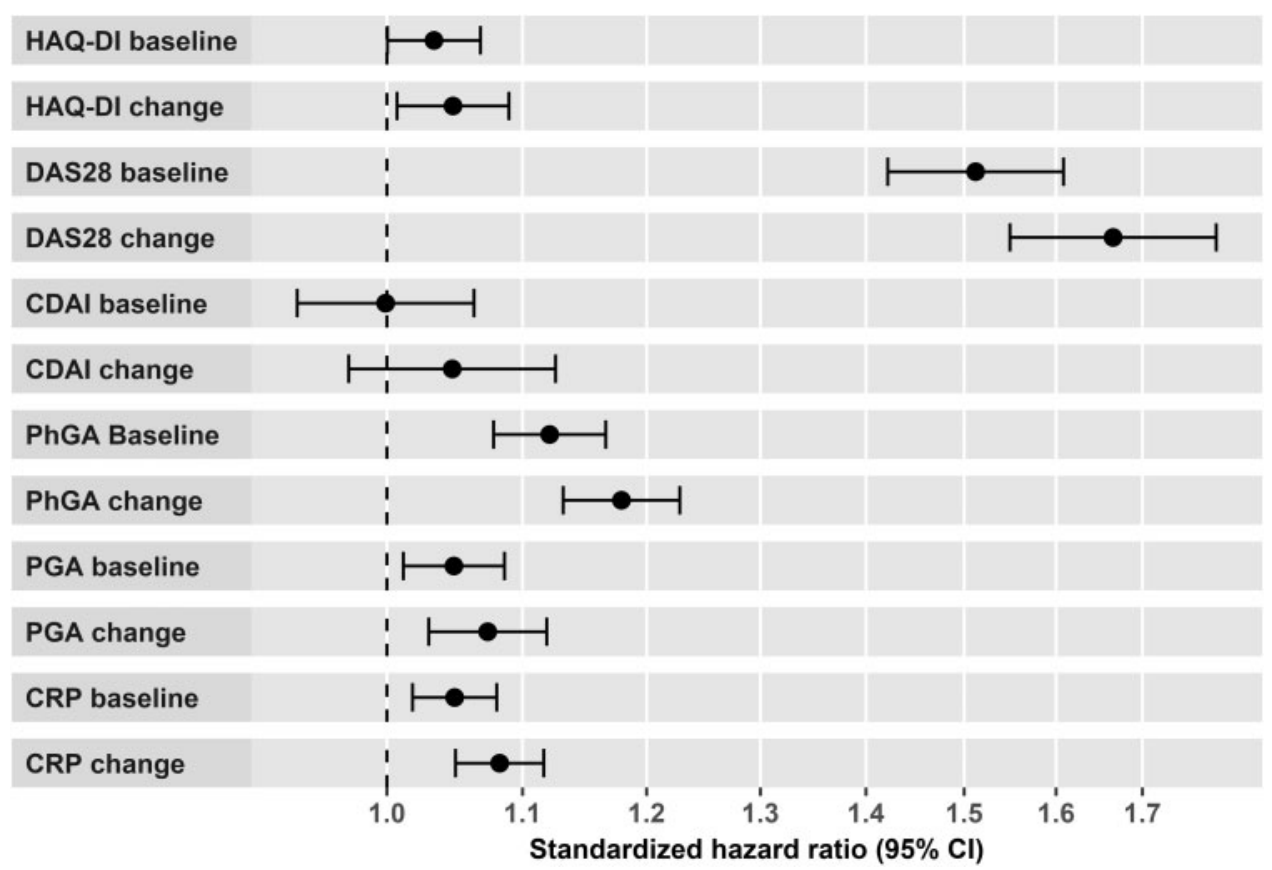

The standardized hazard ratios are adjusted for age, gender, education, smoking, cardiovascular comorbidity, disease duration, seropositivity, number of previously used bDMARDs, presence and type of concomitant csDMARDs, presence of glucocorticoid, difference between tender and swollen joint count and stratified by patient year of treatment initiation and country of register. bDMARD: biological DMARD; CDAl: Clinical Disease Activity Index; csDMARD: conventional synthetic DMARD; DAS28: DAS 28 joints; HAQ-DI: HAQ-Disability Index; PhGA: physician global assessment; PGA: patient global assessment.

subcutaneously [14], it becomes more important that patients get involved in treatment decisions, as shared decision making has been shown to improve drug adherence in RA $[15,16]$. In this light, the results of this study may be a call to action to support a more collaborative patient-physician relationship and foster informed decision on the usefulness of treatment.

PGA was less predictive of drug discontinuation in our study when disease activity scores and PhGA were entered in the model. However, on a positive note, the fact that, when a DAS28 without PGA was used, PGA had almost a similar effect than PhGA suggests that $P G A$ is taken into account in the decision to discontinue the drug. PGA is associated with disease activity, but seems to also measure other aspects such as structural damage and factors not necessarily linked to RA [17]. Although PGA has several advantages, such as easy implementation and high face validity, there is no standardized definition and different responses may be obtained depending on how the question is formulated. As our study is a collaborative register study in clinical settings, there was no possibility to control for this.

An alternative explanation of our results may be linked to the fact that some of the variables are components of the others. For example, some forms of the DAS28 computation include the PGA, making the individual PGA variable less likely to carry much additional information. This was corroborated by the observation that when using a form of DAS28 without PGA, the effect of PGA on discontinuation increased. However, the variance inflation factors were all relatively small $(<5)$ suggesting that these covariates nevertheless explained a differential part of the outcome variability. Considering baseline characteristics, the fact that younger patients and patients with shorter disease duration have greater hazards of discontinuation may be linked to their different expectations than in older patients, with longer disease duration. But it may also warrant further investigations, as this association has not been consistently reported, some studies reporting no effect of age [3] or disease duration [18, 19] and others higher discontinuation with older age $[6,19,20]$ and longer disease duration [3]. As in a univariable model, age and disease duration were non-significant (data not shown), this protective effect of these variables on discontinuation could be due to the adjustment of the model with both baseline and evolution of disease activity. It raises the question of the appropriate management of confounding bias in observational studies, which may lead to paradoxical effect (e.g. the protective effect of obesity in RA) [10]. We found higher discontinuation in females, as described in previous studies [3]. Possible explanations may be that female patients have lower response to 
CsDMARD and TNFi, more subjective pain or that they may be less aggressively treated than men [21-23]. Smoking, which is known to be associated with a higher disease activity, increased risk of extra-articular complications and higher rates of seropositivity, was associated with lower retention, which is in line with previous studies $[6,24]$.

Seropositivity was not associated with higher retention for patients in our study in any of the treatment, which is consistent with the current literature for TNFi and TCZ [8, 25]. However, abatacept has been associated with greater retention in seropositive patients in some studies [18, 26]. Higher numbers of previous bDMARDs $[5,8,27]$ and glucocorticoids at baseline [3] were associated with shorter retention and are probably proxy markers for patients who are more difficult to treat. The protective effect of concomitant csDMARDs is well known with TNFi [2, 4, 6], and has also been described for ABA [28]. For TCZ, the literature is inconsistent, with some studies suggesting higher hazards of retention [29] and other no difference in retention [8] when concomitant csDMARDs are prescribed.

A limitation of our study is that we could not adjust for 'frailty', which has been associated with drug survival in TNFi [4]. Another limitation is the observational nature of the study, with relatively long intervals between assessments, which could lead to missing meaningful changes in disease outcomes and the absence of evaluation of patient adherence. Finally, we did not adjust for other comorbidities than cardiovascular disease. Strengths are the large sample size, the long follow-up and the multiple adjustment for confounding.

In conclusion, we found that the two strongest associations with drug discontinuation of bDMARDs in RA patients were the change in disease activity assessment (DAS28) followed by its baseline value. Importantly, even though DAS28 is considered a less suitable indicator of disease activity than CDAI with IL-6 inhibitors, considering their major effects on inflammatory markers, change of DAS28 was a stronger predictor of discontinuation of bDMARDs than change of CDAl even in TCZ patients. PhGA change was the next strongest predictor of discontinuation, suggesting that the decision to stop bDMARDs is based more on the physician's assessment of disease activity than on the patient's perception of disease severity, which raises some concerns on shared decision making and on the possible implication of the patient's feeling that his or her voice is not often enough taken into account on his or her adherence to the new therapy. Current treatment guidelines insist on shared decision making; however, our results suggest that, currently, the patient's input only secondarily influenced the decision to maintain, or not, bDMARD therapy. More efforts need to be made to include the patient's voice in important treatment decisions, such as continuing, or not, their current bDMARD.

\section{Acknowledgements}

The authors thank all the health professionals and patients who participated in the registers.
Funding: The study is investigator initiated and supported by unrestricted research grants from Bristol Myers-Squibb and Roche. Funding sources had no role in the design of this study, its execution, analyses, interpretation of the data, or decision to submit results.

Disclosure statement: K.L.: reports an unrestricted research grant from AbbVie and non-financial support from Pfizer, outside the submitted work. D.M.: none declared. D.A.-R.: none declared. F.I.: has received speaker or consultant fees from AbbVie, BMS, Celgene, Pfizer, Roche, Novartis and MSD, outside this work. C.C.: has received speaker and consulting fees from AbbVie, Amgen, BMS, Egis, MSD, Pfizer, Roche, Sanofi and UCB. E.K.K.: none declared. T.K.K. has received fees for speaking and/or consulting from AbbVie, BMS, Celltrion, Eli Lilly, Hikma, Hospira, MSD, Mylan, Orion Pharma, Pfizer, Roche, Sandoz, Sanofi and UCB and received research funding to Diakonhjemmet Hospital from AbbVie, BMS, MSD, Pfizer, Roche and UCB. K.P. has received speaker fess from UCB, MSD, BMS, AbbVie, Roche, Amgen, Egis and Pfizer. M.P.-S.: has received consultant and/or speaker fees from AbbVie, Janssen, Lilly, MSD and Sanofi. M.J.S.: has received fees for speaking and/or consulting from AbbVie, Biogen, Roche, Pfizer and Janssen. C.G. has received fees for speaking and/or consulting from AbbVie, BMS, Roche, Pfizer, Celgene, MSD, Janssen Cilag, Amgen and $U C B$, and received research funding from Roche, AbbVie, MSD and Pfizer. A.F. has received consultancies or honoraria from Abbvie, AB2 Bio, BMS, MSD, Pfizer, Roche and UCB and institutional, unrestricted research grants from Pfizer and BMS. D.S.C. has received consulting fees from BMS, Pfizer, AB2 Bio and Janssen.

Clinical work in the Czech Republic was partially supported by the project from the Ministry of Health for conceptual development of research organization MZ00023728 (Institute of Rheumatology). NOR-DMARD was previously supported with research funding to Diakonhjemmet Hospital from AbbVie, BMS, MSD/ Schering-Plough, Pfizer/Wyeth, Roche and UCB. Reuma.pt is supported by unrestricted grants from Abbvie, Biogen, Celgene, MSD, Roche, Sanofi and Pfizer. BIOBADASER has received funding from Fundacion Española de Reumatología, the Spanish Medicines and Health Products Agency (Agencia Española del Medicamento y Productos Sanitarios) and equal grants from pharmaceutical companies (AbbVie, Pfizer, Roche, Schering-Plough and BMS). Swiss Clinical Quality Management in Rheumatic Diseases (SCQM) database is sponsored by public and industrial support (http://scqm.ch/en/sponsoren/). The Romanian Registry of Rheumatic Diseases uses unrestricted grants from AbbVie, Pfizer, MSD, Roche, UCB and BMS.

\section{Supplementary data}

Supplementary data are available at Rheumatology online. 


\section{References}

1 Smolen JS, Landewé R, Bijlsma J et al. EULAR recommendations for the management of rheumatoid arthritis with synthetic and biological disease-modifying antirheumatic drugs: 2016 update. Ann Rheum Dis 2017;76:960-77.

2 Pan SMD, Dehler S, Ciurea A et al. Comparison of drug retention rates and causes of drug discontinuation between anti-tumor necrosis factor agents in rheumatoid arthritis. Arthritis Care Res 2009;61:560-8.

3 Souto A, Maneiro JR, Gómez-Reino JJ. Rate of discontinuation and drug survival of biologic therapies in rheumatoid arthritis: a systematic review and meta-analysis of drug registries and health care databases. Rheumatol 2016;55:523-34.

4 Neovius M, Arkema EV, Olsson $\mathrm{H}$ et al. Drug survival on TNF inhibitors in patients with rheumatoid arthritis comparison of adalimumab, etanercept and infliximab. Ann Rheum Dis 2015;74:354-60.

5 Gabay C, Riek M, Scherer A, Finckh A. Effectiveness of biologic DMARDs in monotherapy versus in combination with synthetic DMARDs in rheumatoid arthritis: data from the Swiss Clinical Quality Management Registry. Rheumatol 2015;54:1664-72.

6 Ramiro S, Landewe R, van der Heijde D et al. Discontinuation rates of biologics in patients with rheumatoid arthritis: are TNF inhibitors different from nonTNF inhibitors? RMD Open 2015;1:e000155.

7 Courvoisier DS, Alpizar-Rodriguez D, Gottenberg JE et al. Rheumatoid arthritis patients after initiation of a new biologic agent: trajectories of disease activity in a large multinational cohort study. EBioMedicine 2016;11:302-6.

8 Lauper K, Nordström DC, Pavelka K et al. Comparative effectiveness of tocilizumab versus TNF inhibitors as monotherapy or in combination with conventional synthetic disease modifying antirheumatic drugs in patients with rheumatoid arthritis after the use of at least one biological disease modify. Ann Rheum Dis 2018;77:1276-82.

9 Nüßlein HG, Alten R, Galeazzi M et al. Prognostic factors for abatacept retention in patients who received at least one prior biologic agent: an interim analysis from the observational, prospective ACTION study. BMC Musculoskelet Disord 2015;16:176

10 Michelsen B, Kristianslund EK, Hammer HB et al. Discordance between tender and swollen joint count as well as patient's and evaluator's global assessment may reduce likelihood of remission in patients with rheumatoid arthritis and psoriatic arthritis: data from the prospective multicentre NOR-DMARD study. Ann Rheum Dis 2017;76:708-11.

11 Smolen JS, Aletaha D. Interleukin-6 receptor inhibition with tocilizumab and attainment of disease remission in rheumatoid arthritis: the role of acute-phase reactants. Arthritis Rheum 2011;63:43-52.

12 Mena-Vazquez N, Manrique-Arija S, Yunquera-Romero L et al. Adherence of rheumatoid arthritis patients to biologic disease-modifying antirheumatic drugs: a cross-sectional study. Rheumatol Int 2017;37:1709-18.

13 Li P, Blum MA, Von Feldt J, Hennessy S, Doshi JA. Adherence, discontinuation, and switching of biologic therapies in Medicaid enrollees with rheumatoid arthritis. Value Health 2010;13:805-12.

14 Chaudhari K, Rizvi S, Syed BA. Rheumatoid arthritis: current and future trends. Nat Rev Drug Discov 2016;15:305-6.

15 Lofland J, Johnson P, Ingham M et al. Shared decisionmaking for biologic treatment of autoimmune disease: influence on adherence, persistence, satisfaction, and health care costs. Patient Prefer Adherence 2017;11:947-58.

16 Mathews AL, Coleska A, Burns PB, Chung KC. Evolution of patient decision-making regarding medical treatment of rheumatoid arthritis. Arthritis Care Res 2016;68:318-24.

17 Nikiphorou E, Radner H, Chatzidionysiou K et al. Patient global assessment in measuring disease activity in rheumatoid arthritis: a review of the literature. Arthritis Res Ther 2016;18:251.

18 Gottenberg JE, Courvoisier DS, Hernandez MV et al. Brief Report: association of rheumatoid factor and anti-citrullinated protein antibody positivity with better effectiveness of abatacept: results from the Pan-European Registry Analysis. Arthritis Rheumatol 2016;68:1346-52.

19 Kristensen LE, Saxne T, Nilsson J-A, Geborek P. Impact of concomitant DMARD therapy on adherence to treatment with etanercept and infliximab in rheumatoid arthritis. Results from a six-year observational study in southern Sweden. Arthritis Res Ther 2006;8:R174

20 Sakai $R$, Tanaka M, Nanki T et al. Drug retention rates and relevant risk factors for drug discontinuation due to adverse events in rheumatoid arthritis patients receiving anticytokine therapy with different target molecules. Ann Rheum Dis 2012;71:1820-6.

21 Jawaheer D, Messing S, Reed G et al. Significance of sex in achieving sustained remission in the consortium of rheumatology researchers of north america cohort of rheumatoid arthritis patients. Arthritis Care Res 2012;64:1811-8.

22 Hyrich KL, Watson KD, Silman AJ et al. Predictors of response to anti-TNF-alpha therapy among patients with rheumatoid arthritis: results from the British Society for Rheumatology Biologics Register. Rheumatology 2006;45:1558-65.

23 Sokka T, Toloza S, Cutolo M et al. Women, men, and rheumatoid arthritis: analyses of disease activity, disease characteristics, and treatments in the QUEST-RA Study. Arthritis Res Ther 2009;11:R7.

24 Söderlin MK, Petersson IF, Geborek P. The effect of smoking on response and drug survival in rheumatoid arthritis patients treated with their first anti-TNF drug. Scand J Rheumatol 2012;41:1-9.

$25 \mathrm{Lv}$ Q, Yin Y, Li X et al. The status of rheumatoid factor and anti-cyclic citrullinated peptide antibody are not associated with the effect of anti-TNF a agent treatment in patients with rheumatoid arthritis: a meta-analysis. PLoS One 2014;9:e89442.

26 Finckh A, Courvoisier D, Gottenberg J-E et al. FRI0221 Does seropositivity influence differentially drug discontinuation of biologic antirheumatic agents with non-antiTNF mode of action? Ann Rheum Dis 2017;76:doi:10.1136/annrheumdis-2017-eular.6006. 
27 Gomez-Reino JJ, Carmona L, Erra A et al. Switching TNF antagonists in patients with chronic arthritis: an observational study of 488 patients over a four-year period. Arthritis Res Ther 2006;8:R29.

28 Truchetet ME, Poursac N, Barnetche T et al. Abatacept monotherapy compared with abatacept plus diseasemodifying anti-rheumatic drugs in rheumatoid arthritis patients: data from the ORA registry. Arthritis Res Ther 2016;18:72.

29 Gabay C, Riek M, Hetland ML et al. Effectiveness of tocilizumab with and without synthetic disease-modifying antirheumatic drugs in rheumatoid arthritis: results from a European collaborative study. Ann Rheum Dis 2016;75:1336-42.

\section{Clinical vignette}

\section{Colchicine gastrotoxicity in a patient with chronic kidney disease}

A 72-year-old man presented with upper gastrointestinal bleeding. He had hyperlipidaemia, gout and chronic kidney disease (calculated glomerular filtration rate $22 \mathrm{ml} / \mathrm{min} / 1.73$ $\mathrm{m}^{2}$ ). Oesophagogastroduodenoscopy showed antral gastritis with erosions and duodenal ulcer. Antral biopsy showed moderate chronic inflammation of gastric mucosa, with numerous mitoses in ring-like configuration (Fig. 1) and apoptotic bodies in the epithelial cells. The findings were compatible with colchicine toxicity [1]. The patient revealed that he had been taking colchicine $0.5 \mathrm{mg}$ twice daily intermittently in the past 6 months for gouty attacks. He was treated with proton pump inhibitor and iron supplement. Haemoglobin level improved from $9 \mathrm{~g} / \mathrm{dl}$ on admission to $12.5 \mathrm{~g} / \mathrm{dl}$ at post-discharge clinic review. Colchicine binds to tubulin to form the poorly reversible colchicine-tubulin complex. The complex blocks the assembly and polymerization of microtubules, thus disrupting cell mitosis [2]. The effect is more pronounced in cells with high proliferative rates. Aside from gastrointestinal toxicity, neuropathy, myopathy and bone marrow suppression have been reported as toxic effects of colchicine. Risk factors for colchicine toxicity include renal impairment, hepatic dysfunction and co-administration of CYP3A4 inhibitors. Colchicine should be avoided in patients with advanced chronic kidney disease (glomerular filtration rate $<30 \mathrm{ml} /$ $\min / 1.73 \mathrm{~m}^{2}$ ).
Rheumatology 2019;58:2229

doi:10.1093/rheumatology/kez178 Advance Access publication 5 May 2019

FIG. 1 Histology of colchicine gastrotoxicity

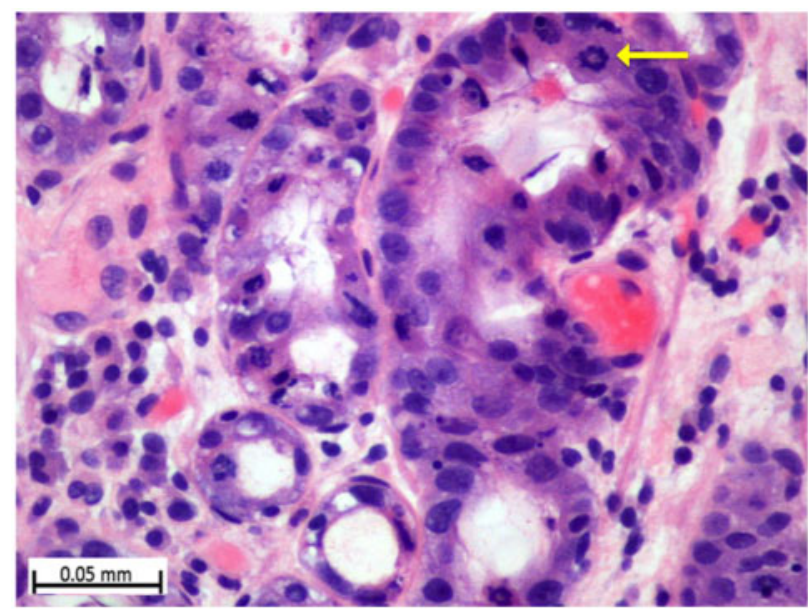

Haematoxylin and eosin stain of antral biopsy: moderate chronic inflammation of gastric mucosa, with mitosis in ring-like configuration (arrow).

(c) The Author(s) 2019. Published by Oxford University Press on behalf of the British Society for Rheumatology. All rights reserved. For permissions, please email: journals.permissions@oup.com 